Pacific Journal of Mathematic 


\title{
BOUNDED ANALYTIC FUNCTIONS ON UNBOUNDED COVERING SURFACES
}

\author{
Shigeo SegawA
}

\begin{abstract}
Let $R$ be an unbounded finite sheeted convering surface over an open Riemann surface with an exhaustion condition. In this paper, the necessary and sufficient condition in order that $H^{\infty}(R)$ separates the points of $R$ is given in term of branch points, where $H^{\circ}(R)$ is the algebra of bounded analytic functions on $R$.
\end{abstract}

A covering surface $R$ over a Riemann surface $G$ is said to be unbounded if for any continuous curve $\lambda ; z=z(t)(0 \leqq t \leqq 1)$ in $G$ and any point $p_{0}$ in $R$ with $\pi\left(p_{0}\right)=z(0)$ there exists a continuous curve $A ; p=p(t)(0 \leqq t \leqq 1)$ in $R$ such that $p(0)=p_{0}$ and $z(t)=$ $\pi \circ p(t)(0 \leqq t \leqq 1)$, where $\pi$ is the projection of $R$ onto $G$. For an unbounded covering surface $R$ over $G$, the number of points of $\pi^{-1}(z)$ is constant $\leqq \infty$ for every $z \in G$ where branch points are counted repeatedly according to their orders. If such a number $n$ is finite, $R$ is said to be $n$-sheeted.

In [2], Selberg proved the following: Let $R$ be an unbounded $n$-sheeted covering surface over the unit disk $|z|<1$ and $\left\{\zeta_{k}\right\}$ the projections of branch points with the order of branching $n_{k}$ over $\zeta_{k}$. Let $z_{0}$ be a point in the unit disk over which there exist no branch points of $R$. Then there exists a single valued bounded analytic function $f$ on $R$ such that $f$ takes distinct values at any two points over $z_{0}$ if and only if $\Sigma n_{k} g\left(\zeta_{k}, z_{0}\right)<\infty$, where $g\left(\cdot, z_{0}\right)$ is the Green's function on $|z|<1$ with pole at $z_{0}$. Yamamura [5] extended the above result to the case where base surfaces are finitely connected plane regions.

On the other hand, Stanton [3] gave another proof of the above Selberg theorem using the Widom results [4]. The purpose of this paper is, by using the Widom-Stanton approach, to establish a result generalizing the Yamamura, and hence the Selberg, theorem to the case where the base surface $|z|<1$ is replaced by certain surfaces which may be of infinite connectivity and genus.

1. Let $R$ be an open Riemann surface of hyperbolic type and $g_{R}(\cdot, p)$ the Green's function on $R$ with pole at $p$. Denote by $H^{\infty}(R)$ the algebra of single valued bounded analytic functions on $R$. For any $\alpha>0$, set $R_{\alpha}=R(\alpha, p)=\left\{q \in R ; g_{R}(q, p)>\alpha\right\}$. It is easily seen that, for each $\alpha, R_{\alpha}$ is connected and $R-R_{\alpha}$ has no compact components. Suppose that each $R_{\alpha}$ is relatively compact in $R$. The 
surface $R$ with this property is referred to as being regular. Let $\beta_{R}(\alpha)=\beta_{R}(\alpha, p)$ be the first Betti number of $R_{\alpha}$. Consider the quantity

$$
m(R)=m(R, p)=\exp \left\{-\int_{0}^{\infty} \beta_{R}(\alpha) d \alpha\right\} .
$$

Widom proved that if $m(R)>0$, then $H^{\infty}(R)$ separates the points of $R$, i.e., for any two distinct points $p$ and $q$ in $R$, there exists an $f \in H^{\infty}(R)$ such that $f(p) \neq f(q)$; it is also shown that $m(R)>0$ does not depend on the choice of points $p$ in $R$. (See [3] and [4].)

2. Hereafter, we suppose that $G$ is an open Riemann surface of hyperbolic type and $R$ is an unbounded $n$-sheeted covering surface over $G$. Then $R$ is also hyperbolic. Let $g_{G}\left(\cdot, z_{0}\right)$ be the Green's function on $G$ with pole at $z_{0} \in G$. Suppose that $G$ is regular and satisfies the condition

$$
\int_{0}^{\infty} \beta_{G}(\alpha) d \alpha<\infty
$$

where $\beta_{G}(\alpha)=\beta_{G}\left(\alpha, z_{0}\right)$ is the first Betti number of $G_{\alpha}=G\left(\alpha, z_{0}\right)=$ $\left\{z \in G ; g_{G}\left(z, z_{0}\right)>\alpha\right\}$. Then, $R$ is also regular.

THEOREM. Under the assumption stated above, the following four conditions are equivalent by pairs:

(i) $m(R)>0$;

(ii) $H^{\infty}(R)$ separates the points of $R$;

(iii) for any $z_{0} \in G-\left\{\zeta_{k}\right\}$, where $\left\{\zeta_{k}\right\}$ is the set of projections of branch points of $R$, there exists an $f$ in $H^{\infty}(R)$ such that $f$ takes distinct values at any two points of $R$ over $z_{0}$;

(iv) $\Sigma n_{k} g_{G}\left(\zeta_{k}, z_{0}\right)<\infty$ for $z_{0} \in G-\left\{\zeta_{k}\right\}$, where $n_{k}$ is the order of branching over $\zeta_{k}$.

Since (i) $\rightarrow$ (ii) has been proved, we only have to show (ii) $\rightarrow$ (iii), (iii) $\rightarrow$ (iv), and (iv) $\rightarrow$ (i).

3. Proof of (ii) $\rightarrow$ (iii). Let $\pi$ be the projection of $R$ onto $G$ and set $\pi^{-1}\left(z_{0}\right)=\left\{p_{1}, \cdots, p_{n}\right\}$ (distinct points) for $z_{0} \in G-\left\{\zeta_{k}\right\}$. Since $H^{\infty}(R)$ separates the points of $R$, there exists an $f_{i j}$ in $H^{\infty}(R)$ such that $f_{i j}\left(p_{i}\right) \neq f_{i j}\left(p_{j}\right)$, for any pair $(i, j)$ with $i \neq j$ and $1 \leqq i, j \leqq n$. We set

$$
F_{i}=\prod_{\substack{1 \leqq j \leqq n \\ j \neq i}}\left(f_{i j}-f_{i j}\left(p_{j}\right)\right) \quad(1 \leqq i \leqq n)
$$

and 


$$
f=\sum_{i=1}^{n} c_{i} F_{i}
$$

for suitable constants $c_{i}$ specified below. Observe that $f \in H^{\infty}(R)$. We can choose constants $c_{i}$ so as to satisfy $f\left(p_{i}\right) \neq f\left(p_{j}\right)$ for any $i \neq j$.

Proof of (iii) $\rightarrow$ (iv). Let $z_{0}$ be an arbitrary point in $G-\left\{\zeta_{k}\right\}$ and $f$ a function in $H^{\infty}(R)$ such that $f$ takes distinct values at any two points over $z_{0}$. Then, by the well known argument of algebroidal functions, it is seen that $f$ satisfies the irreducible equation

$$
f^{n}+g_{1}(z) f^{n-1}+\cdots+g_{n}(z)=0
$$

where $g_{1}(z), \cdots, g_{n}(z)$ are in $H^{\infty}(G)$. Let $D(z)$ be the discriminant of this equation. Observe that $D(z)$ is in $H^{\infty}(G)$, vanishes at every point in $\left\{\zeta_{k}\right\}$, and does not vanish at $z_{0}$. Hence, by the Lindelö principle (cf. [1]), we conclude

$$
\sum n_{k} g_{G}\left(\zeta_{k}, z_{0}\right)<\infty
$$

Proof of (iv) $\rightarrow(\mathrm{i})$. Let $z_{0}$ be a point in $G-\left\{\zeta_{k}\right\}$ and $p_{0}$ a point in $R$ with $\pi\left(p_{0}\right)=z_{0}$. We set

$$
R_{\alpha}=R\left(\alpha, p_{0}\right)=\left\{p \in R ; g_{R}\left(p, p_{0}\right)>\alpha\right\}
$$

and

$$
V_{\alpha}=\{p \in R ; h(p)>\alpha\}
$$

where $h(p)=g_{G}\left(\pi(p), z_{0}\right)$. Denote by $\beta_{R}(\alpha)$ and $\gamma(\alpha)$ the first Betti numbers of $R_{\alpha}$ and $V_{\alpha}$, respectively. We fix $\alpha_{0}(>0)$ such that $V_{\alpha_{0}}$ is connected. Then, $V_{\alpha}$ is also connected for every $\alpha \leqq \alpha_{0}$. By the maximum principle, $h(p) \geqq g_{R}\left(p, p_{0}\right)$, and therefore $V_{\alpha} \supset R_{\alpha}$. Also, by the maximum principle, $V_{\alpha}-R_{\alpha}$ has no relatively compact components in $V_{\alpha}$. Therefore

$$
\gamma(\alpha) \geqq \beta_{R}(\alpha)
$$

Consider each $\alpha$ with $0<\alpha \leqq \alpha_{0}$ such that there exist no branch points of $R$ on $\partial V_{\alpha}$ and no critical points of $g_{G}\left(z, z_{0}\right)$ on $\partial G_{\alpha}$, where $\partial V_{\alpha}$ and $\partial G_{\alpha}$ are the boundaries of $V_{\alpha}$ and $G_{\alpha}$, respectively, and $G_{\alpha}=\left\{z \in G ; g_{G}\left(z, z_{0}\right)>\alpha\right\}$. Let $\hat{V}_{\alpha}$ and $\hat{G}_{\alpha}$ be the doubles of $V_{\alpha}$ and $G_{\alpha}$, respectively. Then, since $\hat{V}_{\alpha}$ can be considered as an unbounded $n$-sheeted covering surface over the compact surface $\hat{G}_{\alpha}$, by the Riemann-Hurwitz and Euler-Poincaré formulas,

$$
2(1-\gamma(\alpha))=2\left(1-\beta_{\alpha}(\alpha)\right) n-2 b(\alpha)
$$


where $\beta_{G}(\alpha)$ is the first Betti number of $G_{\alpha}$ and $b(\alpha)$ is the total sum of the branching order of branch points over $G_{\alpha}$. Thus

$$
\gamma(\alpha)=\beta_{G}(\alpha) n+b(\alpha)-n+1 .
$$

Observe that the set of $\alpha$ such that there exist branch points of $R$ on $\partial V_{\alpha}$ or critical points of $g_{G}\left(z, z_{0}\right)$ on $\partial G_{\alpha}$ is isolated. Hence, from (1) and (2), it follows that

$$
\begin{aligned}
& \int_{0}^{\alpha_{0}} \beta_{R}(\alpha) d \alpha \leqq \int_{0}^{\alpha_{0}} \gamma(\alpha) d \alpha \\
& =n \int_{0}^{\alpha_{0}} \beta_{G}(\alpha) d \alpha+\int_{0}^{\alpha_{0}} b(\alpha) d \alpha+O(\mathbf{1}) .
\end{aligned}
$$

Observe that

$$
\int_{0}^{\alpha_{0}} b(\alpha) d \alpha=\sum_{\zeta_{k} \in G-G_{\alpha}} n_{k} g_{G}\left(\zeta_{k}, z_{0}\right) .
$$

Therefore, by the assumption,

$$
\int_{0}^{\alpha_{0}} b(\alpha) d \alpha<\infty
$$

and also by the assumption

$$
\int_{0}^{\alpha_{0}} \beta_{G}(\alpha) d \alpha<\infty .
$$

From (3), (4), and (5), it follows that

$$
\int_{0}^{\infty} \beta_{R}(\alpha) d \alpha=\int_{0}^{\alpha_{0}} \beta_{R}(\alpha) d \alpha+O(1)<\infty,
$$

i.e.,

$$
m(R)=\exp \left\{-\int_{0}^{\infty} \beta_{R}(\alpha) d \alpha\right\}>0 .
$$

ACKNOWLEDGMENT. The author wishes to express his thanks for helpful suggestions to Professor M. Nakai.

\section{REFERENCES}

1. M. Heins, Lindelöf principle, Ann. of Math., (2) 61 (1955), 440-473.

2. H. L. Selberg, Ein Satz über beschränkte endlichvieldeutige analytische funktionen, Comm. Math. Helv., 9 (1937), 104-108.

3. C. M. Stanton, Bounded analytic functions on a class of Riemann surfaces, Pacific J. Math., 59 (1975), 557-565.

4. H. Widom, $\mathscr{H}_{p}$ sections of vector bundles over Riemann surfaces, Ann. of Math.,

(2) 94 (1971), 304-324. 
5. Y. Yamamura, On the existence of bounded analytic functions, Sc. Rep. T. K. D. Sect. A, 10 (1969), 88-102.

Received November 4, 1977.

Daido Institute of Technology

Daido, Minami, Nagoya 457

JAPAN 



\section{PACIFIC JOURNAL OF MATHEMATICS}

EDITORS

RICHARD ARENS (Managing Editor)

University of California

Los Angeles, California 90024

C. W. Curtis

University of Oregon

Eugene, OR 97403

C. C. MOORE

University of California

Berkeley, CA 94720
J. DUGUNDJI

Department of Mathematics University of Southern Californı Los Angeles, California 90007

R. Finn and J. Milgram Stanford University Stanford, California 94305

\section{ASSOCIATE EDITORS}

E. F. BECKENBACH

B. H. NEUMANN

F. WOLF

K. YoSHIDA

\section{SUPPORTING INSTITUTIONS}

UNIVERSITY OF BRITISH COLUMBIA
CALIFORNIA INSTITUTE OF TECHNOLOGY
UNIVERSITY OF CALIFORNIA
MONTANA STATE UNIVERSITY
UNIVERSITY OF NEVADA, RENO
NEW MEXICO STATE UNIVERSITY
OREGON STATE UNIVERSITY
UNIVERSITY OF OREGON

UNIVERSITY OF SOUTHERNEALIFORNIA STANFORD UNIVERSITY UNIVERSITY OF HAWAII UNIVERSITY OF TOKYO UNIVERSITY OF UTAH WASHINGTON STATE UNIVERSITY UNIVERSITY OF WASHINGTON 


\section{Pacific Journal of Mathematics \\ Vol. 79 , No. 1 \\ May, 1978}

Teófilo Abuabara, A remark on infinitely nuclearly differentiable

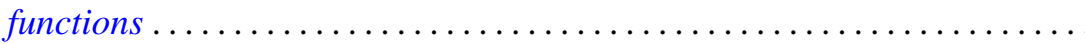

David Fenimore Anderson, Projective modules over subrings of $k[X, Y]$

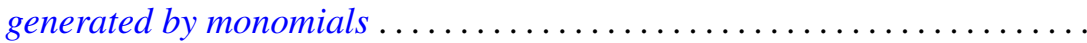

Joseph Barback and Thomas Graham McLaughlin, On the intersection of

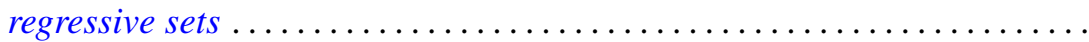

Murray Bell, John Norman Ginsburg and R. Grant Woods, Cardinal inequalities for topological spaces involving the weak Lindelof

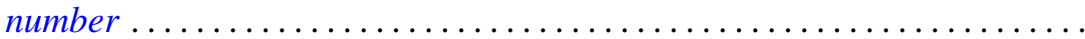

Laurence Richard Boxer, The space of ANRs of a closed surface ............

Zvonko Cerin, Homotopy properties of locally compact spaces at

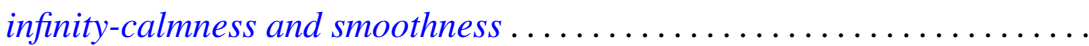

Isidor Fleischer and Ivo G. Rosenberg, The Galois connection between partial functions and relations..................................

John R. Giles, David Allan Gregory and Brailey Sims, Geometrical implications of upper semi-continuity of the duality mapping on a Banach

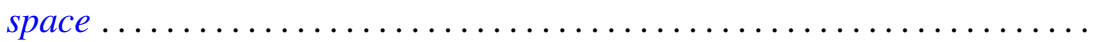

Troy Lee Hicks, Fixed-point theorems in locally convex spaces ............ Hugo Junghenn, Almost periodic functions on semidirect products of transformation semigroups ........................

Victor Kaftal, On the theory of compact operators in von Neumann algebras. II . . . .

Haynes Miller, A spectral sequence for the homology of an infinite

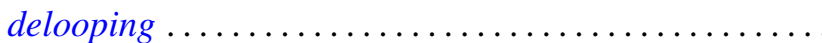

Sanford S. Miller, Petru T. Mocanu and Maxwell O. Reade, Starlike integral operators...

Stanley Stephen Page, Regular FPF rings ...............

Ghan Shyam Pandey, Multipliers for C, 1 summability of Fourier series ...

Shigeo Segawa, Bounded analytic functions on unbounded covering surfaces...

Steven Eugene Shreve, Probability measures and the C-sets of

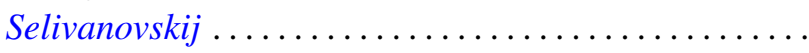

Tor Skjelbred, Combinatorial geometry and actions of compact Lie groups....

Alan Sloan, A note on exponentials of distributions.

Colin Eric Sutherland, Type analysis of the regular representation of a nonunimodular group.

Mark Phillip Thomas, Algebra homomorphisms and the functional

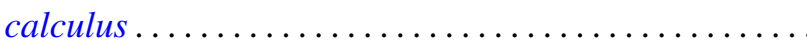

Sergio Eduardo Zarantonello, A representation of $H^{p}$-functions with

$0<p<\infty$. 